\title{
Neurophysiological background for physical therapies in fibromyalgia
}

\author{
R. Casale ${ }^{1}$, F. Atzeni ${ }^{2}$, P. Sarzi-Puttini ${ }^{2}$ \\ ${ }^{1}$ Department of Clinical Neurophysiology \& Pain Rehabilitation Unit, Rehabilitation Institute of Montescano, \\ IRCCS, Foundation Salvatore Maugeri, Pavia, Italy; \\ ${ }^{2}$ Rheumatology Unit, L. Sacco University Hospital, Milan, Italy
}

\section{SUMMARY}

This paper describes the techniques for controlling pain by the physical means that are most widely used clinically, particularly in the case of fibromyalgia. They are grouped on the basis of the physical energy used: mechanical, thermal (including magnetic and electromagnetic), and light (LASER). The main underlying neurophysiological mechanisms are gate activation, the stimulation of descending systems of pain control, and the endogenous opiate system.

Key words: Physical therapies, chronic pain, fibromyalgia, neurophysiology.

Reumatismo, 2012; 64 (4): 238-249

\section{INTRODUCTION}

T he methods so far proposed for controlling pain in patients with fibromyalgia (FM) are essentially based on four components: surgery, drugs (including anesthetics), cognitive-behavioural procedures, and techniques of sensory modulation.

In general, surgery and the destructive means of pain control (rhizotomy, cordotomy, phenolisation and alcohol treatment of ganglia, thermocoagulation of the trigeminal nucleus, etc.) have very restricted indications and various limitations, and rarely lead to adequate and lasting pain control. Furthermore, neurolesive techniques can have disastrous effects, such as anaesthesia dolorosa (1). Although surgical techniques are not indicated for FM, as shown on the American College of Rheumatology website (2), some invasive or minimally invasive treatments have been proposed without any scientific support.

The currently used drugs often have poorly tolerated side effects, but they are capable of reducing pain in most patients, as demonstrated by systematic reviews of the literature. Essentially, the four groups of drugs used for oral administration are antiepileptic agents and membrane stabilisers (gabapentin, pregabalin, etc.) (3); weak and strong opioids in non-oncological pain (ketorolac, tramadol, codeine, morphine, etc.) $(4,5)$; serotoninergic, noradrenergic and combined anti-depressants (venlafaxine, duloxetine) (6); and non-steroidal antiinflammatory drugs alone or in association with rehabilitation strategies (7).

Local anaesthetics are, in practice, membrane stabilisers, but they have particularly useful characteristics and have been found to be useful in managing neuropathic pain (8). The local infiltration of anaesthetics is very frequently used for pain control but, for technical reasons, this strategy is limited to localised pain and anatomically accessible zones. The repeated anaesthetic blockade of trigger points as in FM should have the effect of drastically decreasing the afferent barrage to the spinal cord and, therefore, contribute to reducing the spinal phenomena of wind-up and sensitisation, thus reducing spino-thalamic afferent traffic and perceived pain for a much longer time than the anaesthetic is active. Recently botulin toxin has been shown to have some effect on pain suggesting its potential specific use in myofascial pain (9).

Cognitive-behavioural techniques are extremely important in FM. We will here 
mention only the major results of "short cognitive therapy" in the management of chronic malignant pain, and in some pain disorders frequently seen in rehabilitation settings, such as myofascial pain (10) and complex regional pain syndromes (11). There is insufficient clinical evidence concerning the group of so-called mind-body interventions to support the spread of techniques such as muscle relaxation, meditation, "imagery", hypnosis, tai-chi, qi-gong and yoga, although they are suitable for increasing well-being in the elderly (12).

\section{Sensory modulation by physical energy}

Any type of exteroceptive or proprioceptive stimulation is ultimately transformed into nerve impulses that travel along peripheral nerves to the neuroaxis, and give rise to a whole series of electrical events that are decoded by the central nervous system on the basis of the type, frequency, duration and intensity of the stimulus. Movement (proprioceptive stimulation) and physical therapies (exteroceptive stimulation) therefore activate different mechanisms depending on these parameters. There are basically three systems of pain control: spinal gating (13), descending inhibitory noxious control (DINC) systems (14), and the system of endogenous opiates (15). Extra- synaptic or volume transmission is potentially capable of providing clues to the therapeutic effects of some physical treatments, but it is still largely a subject of research and, although its importance can be sensed, it will not be mentioned any further except as a possible mechanism. Hypothetical metabolic effects, which in any case act as intermediaries between therapeutic stimuli and anti-nociceptive nervous responses, will not be discussed either.

In general, any physical agent that produces a perceivable stimulus can be classified into one of two broad groups: physical therapies that produce non-painful sensations, and those that induce painful or almost painful sensations. The former activate largediameter, myelinated A- $\beta$ afferent nerve fibres, the most important mechanism of pain control in the spinal cord; the latter include types of physical energy that produce painful or at lest intense sensations, and predominantly activate descending control systems such as the DINC and the opiate system. The predominance of one over the other is subject to genetic factors (16), gender and individual factors (17).

On the basis of these premises, and given the specific characteristics of movement and physical therapies, these two forms of sensory modulation will be dealt with separately.

\section{Physical therapies}

Physical therapies are widely used in FM and have a broad range of thermal, electrical, mechanical and other sources (18). Some clinical successes have been reported in the literature, but there is no incontrovertible evidence to support the use of specific physical therapies or specific physical agents in the treatment of pain in general, although there is a sufficient theoretical basis for their use in controlling pain even in FM patients. One general critical issue concerning all physical therapies is the delivered therapeutic dose. All of the reviews of published studies highlight the difficulty of comparing the research results because of the lack of information about the method used and, in particular, the physical characteristics of the stimulus (intensity, frequency, duration, time and site of application).

Another general critical aspect of the use of physical therapies is the lack of a rational combination with drugs. There is solid evidence that drugs can have both negative and positive effects on some physical therapies: e.g. it is known that benzodiazepines negatively influence acupuncture (19), and that serotonin (and therefore serotoninergic anti-depressants) have a potentiating effect (20). With this background, a therapeutic approach based on pain mechanisms seems to be the only way of ensuring the correct clinical use of all of the possible physical therapies for pain $(18,21)$. It is important to remember that, whatever form of energy is applied, this energy is transformed into impulses that travel along a peripheral nerve to the central nervous system, where they have their main effect. It is therefore not so much the type of energy that is im- 
portant, but the neurophysiological target of the stimulation and the possible use of appropriate pharmacological support.

Almost all physical therapies have been applied to FM (18). A traditional classification distinguishes: manual therapy (therapeutic exercise, massage, manipulations, etc.), mechanical therapy (traction, compression, vibration, etc.), superficial heat (dry and humid) and deep heat therapy (short wave and microwave diathermy, ultrasound); cold therapy (cooling sprays, bricks of pre-frozen substances, ice cube massage, hypothermia); and electrical therapy with alternating currents (faradic, sinusoidal, Transcutaneous Electrical Nerve Stimulator (TENS), etc.) or direct currents (galvanic). However, another way of classifying physical therapies, and one that is perhaps closer to the underlying mechanisms of action and concepts set out at the beginning of this paper, is to distinguish the therapies on the basis of the type of energy used: mechanical, thermal, electrical (including magnetotherapy and electromagnetotherapy), and light (LASER).

\section{Mechanical energy}

This group includes manual therapies (joint manipulation, osteopathy, mobilisation), massage, ultrasound, shock waves and vibration. The reason for this choice is that these techniques share the same neurophysiological mechanisms of pain control.

\section{Manipulation-mobilisation}

The rationale underlying the undeniable success of manual therapy in some cases, particularly osteopathy (22) and vertebral manipulation (23), has not changed much over the years (24). The first tentative explanations included fairly empirical concepts that were never demonstrated, such as articular and postural adjustments, restoration of bone alignment, and reduced disc bulging in the case of vertebral manipulation. Subsequently, a conceptual shift led to it being considered that manual therapy was capable of modifying the biology of collagen and muscle, and a generic requirement to maintain joint function was thought to be important to preserve high functional levels throughout life. These are certainly important effects that deserve further investigation, but they do nothing to explain the often exceptional results that manipulation and mobilisation have on pain, nor in which patients or in what types of disorders they have the best results (25).

Any type of manipulation or mobilisation of joint heads is capable of sending a brief but very powerful mechanoreceptor input to the spinal cord through the rich external and proprioceptive innervation of the joints. This input travels along the fast-conducting, large diameter (A- $\beta$ )sensory nerve fibres. The involvement of fast systems of pain control was demonstrated by an elegant experimental model and, in humans, it was shown that a single mobilisation could immediately raise the pressure pain threshold of a muscle of the same metamere as that mobilised (26). Metamerism and a fast onset of effect are characteristics of spinal gating. These neurophysiological findings are indirectly supported by the clinical and empirical idea of the "rule of no pain" (23), according to which any type of vertebral mobilisation must not cause pain. The "rule of no pain" adds the activation of afferent systems not related to nociception to the other typical characteristics of gating. Although some researchers have suggested that manipulation may mediate endorphinergic activity (25), this has never been demonstrated. The reduction in pain induced by endorphin production could be related to a placebo effect (27) and patient expectations (28). The possibility of a placebo-induced release of endorphins is common to all the cognitive behavioural techniques and physical therapies.

\section{Massage}

The hands and manual massage are certainly the oldest therapeutic instrument ever used by man; nevertheless, the physiological basis of the effect of massage has not yet been fully clarified (29) although there seem to be local and reflex action (30) probably related to arousal phenomena. In animals, a mechanoreceptor-transduced tactile stimulus such as suckling during lactation induces the production of the 
neurohormone oxytocin by means of a frequency-dependent mechanism (31). This suggests that appropriate tactile stimuli can have very different actions from classical local stimuli. One of the best known and accredited forms of massage is the "reflex connective tissue massage" or "Bindegewebsmassage" (32), which requires semeiologically precise verification of the type and intensity of the stimulus delivered. Other types of massage that are only based on the sensitivity of the masseur's hands do not enable any type of standardisation or harmonisation of the amount of energy/therapy delivered, and will not be considered here, although it is reasonable to suppose that they may act by means of the same mechanisms. The technique of Teirich-Leube and Dicke has two components: "superficial stimulation" and "deep stimulation"(32). If correctly performed, the first produces a sensation of fluttering by means of stroking, and the second creates the sensation of a sharp cut in the skin. Both sensations are clearly perceptible to patients, who can describe them, thus enabling a physiotherapist to be certain that an adequate mechanical stimulus is being delivered. From a neurophysiological point of view, the fluttering is related to the stimulation of slowly adapting mechanoreceptors and transmitted by A- $\beta$ fibres (33), whereas the (almost painful) cutting sensation, is transmitted by the A- $\Delta$ fibres connected to free nerve endings. It is clear that here there are the conditions for both the activation of spinal gating, and a type of counter-irritation (34) activating the DINC and opiatergic systems.

\section{Vibration}

When a mechanical vibratory stimulus is applied to the tendon of a muscle, it causes a barrage of impulses along the large-diameter Ia afferent nerve fibres, thus mimicking what occurs in normal isometric muscle contraction. From this point of view, vibration and movement share the same afferent mechanism of activating gating (35). Similarly, vibration and massage share afferents mediated by Pacini corpuscles: regardless of whether the source is a me- chanical, pneumatic or "manual" vibrator, when the stimulus supplied is around 40 $\mathrm{Hz}$, the sensation evoked is that of a flutter (36). True sensations of vibration occur at higher frequencies, with optimal receptor responses between 200 and $300 \mathrm{~Hz}$ (37). A vibration stimulus is a very potent generator of sensory afferents, and is capable of inhibiting spinal nociceptive reflexes (38). The vibration-induced activation of gating is frequency related. Vibration has been used with positive effects on pain (39) and movement (40). Like vibration, TENS activates spinal gating, but another similarity between the two is widespread confusion regarding the frequency and amplitude of the stimulus. The use of pneumatic vibrators capable of delivering very high frequency $(200-300 \mathrm{~Hz})$ stimuli, a technology that was confined to physiology laboratories until a few years ago, currently seems to be giving new impetus to the vibratory treatment of pain. Thus the possibility of using mechanical vibratory stimuli in FM relies on the double effect of vibration on fatigue and pain perception (41).

Ultrasounds are high frequency sonic vibrations $(16,000 \mathrm{~Hz}$.) and should therefore theoretically be included in this section concerning vibratory stimuli in general. However, their essential action is to produce heat and increase the temperature of deep tissues; there is no concerning the action of ultrasonic vibrations through mechanoreceptors.

\section{Shock waves}

The stimulus of high-energy acoustic waves generated in various ways (electrodynamically, electromagnetically or piezoelectrically) is transmitted through mechanoreceptors and perceived by subjects as being considerably intense. Shock waves are therefore considered in the group of counter-irritation therapies. The analgesic effect of shock waves involves the activation of the descending mechanisms of pain control (DINC) and the system of endogenous opiates. Although there are no data concerning the type of neuropeptides stimulated by this type of therapy, the effects are probably mediated by $\beta$-endorphins 
rather than dynorphins. Experience in large animals has shown the onset of an analgesic effect $15 \mathrm{~min}$ after the application of the stimulus, thus leading to the hypothesis that other types of rapidly onset analgesic activity my also play a role (42). The albeit slight analgesic activity of extra-corporeal shock waves seems to be confirmed by recent meta-analyses (43).

\section{Thermal energy}

Thermotherapy involves the application of heat or cold for therapeutic purposes, and are both thought to have a direct analgesic effect mediated by mechanisms of counterirritation (44). However, precisely because thermal energy can evoke painful or almost painful sensations, they must be used very close to the threshold of tissue damage. From a theoretical point of view, thermotherapy has many limitations and its use is always related to the patient's choice.

\section{Heat}

Moderate heat lowers the threshold of excitability of mechanoreceptors, thus making them more sensitive (45). Only at a temperature of about $47-49^{\circ} \mathrm{C}$ do mechanoreceptors become less excitable, but these temperatures are neither reached nor tolerates by patients in a clinical setting, especially fibromyalgic patients. Furthermore, the progressive breakdown of cell membranes and enzymes, and their almost total inactivation at such temperatures, could mediate the production of heat shock proteins, which are involved in functions that are essential for cell survival.

Although the marked anti-inflammatory activity of heat therapy in rheumatoid arthritis has been demonstrated (46), there are no studies that have directly correlated this treatment with a reduction in pain. However, heat therapy continues to be widely used in painful bone and joint disorders, as well as low back pain, although the published results are still inconclusive (47). The effects of heat delivered systemically (as in the case of a sauna or steam bath), or locally to the body's surface, are essentially linked to the sphere of subjective well-being and a certain local vasodi- latory action; the data concerning the analgesic mechanism of action and efficacy of these techniques are almost completely anecdotal.

Such sources of heat (like others such as paraffin gloves and heat pads) induce an exogenous increase in surface temperature, but there are other methods of generating heat that raise the temperature of tissues by means of the Joule effect. These methods are endogenous producers of heat based on equipment that generates short waves (Marconi therapy), microwaves (radar therapy and hyperthermia), and diathermy. Obviously, not all of the physical phenomena induced by heat through the Joule effect are therapeutically important, but some local and systemic effects could have a certain biological relevance, particularly local effects on cell metabolism, blood flow and connective tissue. The metabolism of a cell exposed to heat can increase by as much as $13 \%$ with every degree Celsius and, if appropriately channelled, this potent metabolic activity could activate mechanisms of cell self-repair (44). It has not been demonstrated that these metabolic effects are directly related to an effect on pain.

One particular case of the production of endogenous heat is that of diathermy, which is produced by means of "combined capacitive/resistive therapy" at fixed or variable frequencies that can range from 100 to $850 \mathrm{kHz}$. Although it seems that there have already been positive clinical results, there are no published data concerning the percentage success rate of the treatment. In particular, there are no data concerning the specific therapeutic aspects affected by the various frequencies, or concerning which tissues to target, or what disorders to treat.

\section{Cold}

Both heat and cold can increase or decrease the speed of peripheral nerve conduction with a Gaussian relationship (48), and so both could have direct effects on the balance of afferents to the spinal gate, and ultimately contribute to strengthening the gate. Cold can induce reactive vasodilation and the production of inflammatory mediators (49) that could aggravate an underlying 
inflammatory condition. Furthermore, inappropriate exposure to cold leads to the formation of ice crystals that increase the osmolarity of extracellular fluids and cause intracellular dehydration and cell damage (49). Nevertheless, cold has a niche in the short-term control of acute pain, such as dental pain. In this setting, the application of ice to an acupuncture site on the hand ipsilateral or contralateral to the pain can reduce its intensity by $50 \%$ (50) and, in some patients with low back pain, can be superior to TENS (51). The application of cold in the form of massages with ice certainly has a statistically positive action on range of movement and inflammatory oedema in patients with osteoarthritis but, unfortunately, not on pain. Cold is also widely used because its action on neuromuscular spindles causes a clinical reduction in muscle tone (44). However, once again, there are no data concerning any direct influence pain transmission systems.

\section{Electrical energy - electrotherapy}

Electrotherapy covers an innumerable series of techniques based on passing an electrical current through the human body. Although instruments that generate all types of waves and frequencies are available, the currents can basically be divided into direct and alternating currents. Among the latter, TENS is the most widely used and gives the best clinical results, although a systematic analysis of the published does not allow any final conclusions to be drawn because of a lack of information concerning stimulation frequencies (52).

There are essentially two types of TENS in clinical practice, which have different mechanisms of action in relieving pain. High-frequency TENS (100-200 Hz) (TENS-HF) delivered at low intensity (under the level of muscle contraction) mainly acts by recruiting the highly myelinated A- $\beta$ afferents related to spinal gating. It has a rapid onset of action that produces a strong (but not painful) tingling sensation, but has a short-lasting effect that is also limited to the stimulated metamere. TENS-HF does not affect peripheral nerve conduction (53). On the contrary, low-frequency (2-10
$\mathrm{Hz}$ ), high-intensity TENS (34) is a frankly painful stimulus that is always accompanied by intense muscle contraction, and is therefore known as acupuncture-like TENS (TENS-A). It induces analgesia more slowly, but the analgesic effect is longer lasting and extends beyond the treated metamere. If electrical stimulation is applied percutaneously through a needle, the technique is called electroacupuncture, which does not differ from TENS-A except for the lower intensity of the currents that can be used. The effect of such techniques is mainly based on the involvement of opiatergic systems. In humans, the intravenous injection of small doses of the opiate antagonist naloxone can antagonise the analgesia produced by TENS-A, but not that produced by TENS-HF. This has led to the hypothesis that their mechanism of analgesia are different: an opiatergic action for TENS-A but not for TENS-HF, as the analgesia it induces is not reversed by naloxone (54). In vitro experiments using specific antagonists for the different opiate receptor subtypes have confirmed that low-frequency electrical stimulation induces the activation of $\mu$ and $\delta$ receptors and the production of $\beta$-endorphin, and is antagonised by naloxone but, although high-frequency stimulation also induces the activation of endogenous opiates, the analgesia is not sensitive to naloxone because it involves $\kappa$ receptors and dynorphins that are not antagonised by naloxone (41). From a pragmatic point of view, if the aim is to activate all three receptor subtypes $(\mu, \delta$ and $\kappa)$ simultaneously, it would be necessary to combine high- and low-frequency stimuli (41).

It has been ascertained that other neurotransmitters are involved in anti-nociception and, particularly, the analgesic use of electrical currents. It has also been recently shown that GABAergic mediation plays a role in the effects of electrical currents, regardless of their frequency (55) or wave form (56).

One critical parameter is the intensity of stimulation that can be used in man: in vitro experiments are one thing but, in a clinical setting, intensities delivered at low frequencies are no longer tolerated if they 
are combined with high frequencies. Given the complexity of the interactions between spinal gating and the descending systems of pain control, and the differences in their activation, it is clear that any attempt to manipulate them without understanding their mechanisms of action in different diseases, and in the absence of a correct classification of the type of pain to be treated, will lead to disappointing results. One clear demonstration of the importance of a correct diagnosis and the consequent choice of technique has been provided by a meta-analysis showing that, although both TENS-HF and TENS-A have a positive effect on low back pain, there is a clear difference in favour of TENS-A $(45.80 \%$ vs $86.70 \%$ ) (57).

The evolutions of TENS include implanted perineural and subcutaneous stimulators, and techniques in which the electrodes are inserted into the peridural space instead of being placed on or penetrating the skin. The analgesic results of these relatively simple techniques are virtually the same, but it is also possible to obtain therapeutic effects in the case of muscle tone disorders such as spinal spasticity. Other methods of supplying an electrical current through an electro-conductive glove have been recently proposed (TENS-touch mode), a technological revival that has been made possible as better electrical coupling has surpassed the concept of static electrode application. Another recently introduced analgesic electrotherapy is "scrambler therapy", in which the frequencies and intensities are delivered in a "quasi-stochastic" manner. However, no adequate clinical data are available for either technique. Interferential currents have substantially the same analgesic effect as TENS-HF, and act on the same neurophysiological mechanisms (58).

In brief, like those of acupuncture, the results of TENS are determined by the perceived intensity of the stimulation (the sensation of te-chi in traditional Chinese acupuncture) and the frequency of the stimulation (TENS-HF vs TENS-A). Afferent fibres from the periphery arrive at the spinal gate, where different and potent endogenous inhibitory systems can play a modulating role. In animals, different frequencies of stimulation seem to activate specific central nervous system structures and different mechanisms of producing endogenous opiates, with presumably different effects on clinical pain mediated by encephalins, dynorphins or their spinal synergism (41). These different actions could justify the clinical use of alternating bursts of high and low frequency TENS, and also raise unanswered questions about the possible differential production of endogenous opiates induced by other forms of frequency-dependent physical therapy.

The only rational application of direct currents is iontophoresis, which makes it possible to deliver small molecules (such as lidocaine) or more complex molecules into the deep tissues, albeit in an uncontrolled manner. Rapid advances in microelectronics, nanotechnology and the miniaturisation of electromedical devices has opened up the way to new systems that can vehicle drugs, such as fentanyl, and simultaneously optimise the amount of drug released (59) .

\section{Electromagnetic energy}

Magnetotherapy is based on the physical principle of electromagnetic induction: an electrical current passing through any conductor generates a magnetic field, the intensity of which is inversely proportional to the length of the conductor. The literature contains descriptions of the use of low- frequency magnetic fields $(<100 \mathrm{~Hz})$ at intensities of 1-100 Gauss, and high-frequency magnetic fields (up to $20 \mathrm{MHz}$ ) at low intensity (60). Exactly how these induce a clinically appreciable reduction in pain has not yet been clarified.

The biological effects of the application of a magnetic field to cells range from altered cell proliferation to changes in transmembrane ion flow. The cell membrane and its voltage-dependent ion channels seem to be the main points of action (61). Our previous considerations concerning possible differences in the endogenous opiate production stimulated by different frequencies, and the importance of the intensity of the stimulus to evoke spinal gating rather than the production of opiates, also apply to magneto- 
therapy. A pilot study by Markov's group found that this technique was efficacious in uncomplicated low back pain.

The possibility that magnetotherapy might distort the electromagnetic field of cells, and thus influence neurohormonal patterns or mechanisms of volumetric extra-synaptic transport should also be considered. There are data indicating that the former effect does not occur (62), whereas there is no information concerning the latter.

\section{Light energy - LASER}

LASER is the acronym of "light amplification by stimulated emission of radiation". The most important characteristic of this form of energy is the wave length, which conditions the capacity of radiation to cross and be absorbed by tissues, and therefore the type of tissue the radiation reaches and the conditions that can be treated. Another important parameter is power: the greater the power, the greater the energy that can be transferred to deep tissues. One possible classification of the various types of LASER can therefore be based primarily on the wavelength emitted, as a function of the tissues to irradiate, and power rather than the type of technology used (solid-state, gaseous, semi-conductance). The best wavelengths for clinical use are those within the "therapeutic window" of $600-1100 \mathrm{~nm}$. The most modern equipment combines emissions at $810 \mathrm{~nm}$ and $980 \mathrm{~nm}$ or $1064 \mathrm{~nm}$ in order to ensure less scattering by water, haemoglobin and pigments, all of which act as acceptors of the light and prevent the irradiation of tissues. Using these wavelengths at high intensities, tissue layers can be reached that would otherwise be inaccessible. LASER has been accredited as having bio-stimulatory effects by stimulating molecules called photo acceptors (cytochrome $\mathrm{C}$ oxidases) of the intracellular respiratory chain. At cell level, the proposed mechanisms are an effect on the redox state and superoxide anions, and a local micro-thermal effect (63) when lower power in the order of a few micro-watts is used. The currently available high-power equipment (15-25 W) amplifies this effect and spreads it into deep tissues. The effect of LASER on nitric oxide is particularly important because this molecule is involved in the transmission of pain (64).

In relation to the therapeutic effect of LASER on nociception and anti-nociception, it has been demonstrated that exposure of the (sensory) dorsal ganglia inhibits the excitatory action of bradykinin, thus suppressing the transmission of impulses along the primary sensory afferents (65). LASER can also inhibit pro-algogenic substances in free nerve endings in the presence of inflammation (66) and, very interestingly, activate descending pain control systems. One factor that seems to be very important in determining the therapeutic effects of LASER is the method of emission: pulsed ( predominantly photomechanical) or continuous.

As in the case of the other physical therapy techniques, the conclusions emerging from meta-analyses of LASER highlight the lack of information concerning the methods used, the small number of subjects studied, and the need to obtain more data on the effects of different doses. Nevertheless, even the systematic reviews show a degree of concordance about the efficacy of LASER in controlling pain (67). It has recently been demonstrated that LASER can reduce pain and also improve neurophysiological parameters when combined 830 and 1064 $\mathrm{nm}$ high-intensity LASER is used (68).

\section{CONTRAINDICATIONS, WARNINGS, ERRORS AND SIDE EFFECTS}

Every effective pharmacological therapy has contraindications and this universally recognised idea has been simply transferred to physical therapies without making due distinctions. Furthermore, the contraindications to physical therapies given in textbooks range from local conditions that prevent their application, to cancer, pacemakers, tuberculosis, multiple sclerosis, myasthenia, parkinsonism, bleeding disorders, metabolic diseases, pregnancy, etc. There is a degree of concordance in the literature about the contraindications to certain phys- 
ical therapies, such as superficial and deep (ultrasound) thermotherapy, but the data always come from earlier studies and the bibliographical references are very different and not unequivocal (69). This suggests that the contraindications have been "handed down" from one publication to another, in a rather anecdotal manner without any real investigation into the problem. In any case, the conditions contraindicating the use of any given physical therapy should be scrupulously evaluated, and the specific therapies prescribed should be chosen on the basis of published data. Nevertheless, in the absence of a demonstrated cause-effect relationship between the use of a treatment and the appearance of a complication or the worsening of a pre-existing condition, many contraindications seem to be more a medico-legal safeguard than a real problem.

Iatrogenic conditions caused by the wrong choice and/or incorrect application of a technique should also be considered as it goes without saying that these can unfortunately occur, and indeed often do when using the techniques of osteopathy and vertebral manipulation (70).

In order to reduce errors, some real but often ignored contraindications should be considered. In order to work, a physical therapy must provide the central nervous system with useful signals that can be deciphered in terms of the frequency, duration and intensity of the stimulation. Any lesion of the central or peripheral afferent sensory pathways is therefore a potential contraindication that requires careful evaluation because, in the absence of an afferent pathway or the presence of an altered pathway, the stimulus cannot reach its target or, if it does, may transmit distorted (and therefore not useful) or even harmful information. Furthermore, the application of a physical stimulus to an area of anaesthesia or hypoaesthesia is dangerous because, as there is a sensory disorder, it is easy to cause iatrogenic damage by using excessive stimulation. This danger also exists when treating children, the elderly or patients with cognitive deficits who are unable to describe the evoked sensation correctly. There are no reliable published data concerning side effects or statistics to. The best-known clinical side effects are those due to activation of the autonomic nervous system, such as sweating and piloerection, sometimes associated with rotational vertigo, nausea and, in some cases, vomiting. The effects on sympatho-vagal balance, such as those caused by TENS (71), are less clear.

\section{A POSSIBLE CONCLUSION}

All physical therapies are capable of having a clinical, anti-nociceptive action that can be perceived by patients and recorded by physicians $(18,21)$. However, when seeking the pathophysiological basis underlying these undeniable improvements, one is often left floundering in a search for unlikely mechanisms. Systematic reviews have shown that, in practice, there is a substantial lack of data, although it needs to be stressed that not even the results of Cochrane Library reviews are free from criticism (72).

In conclusion, this only apparently severely critical paper has the purpose of clearing the field of the usual "metabolic" theories (proven only in rare cases such as LASER) in order to examine physical therapies from the point of view of the nervous system and its interactions with the neuroendocrine system. The nervous system is a plastic and modifiable place in which all sensory afferents are integrated, with sometimes potentiating and sometimes inhibiting effects. Without these properties, which present throughout our lifetimes, any treatment based on the transmission of sensory inputs to a system would be doomed to failure from the outset, as would physical therapies and the rehabilitation of neurological lesions.

\section{REFERENCES}

1. Lopez BC, Hamlyn PJ, Zakrzewska JM. Stereotactic radiosurgery for primary trigeminal neuralgia: state of the evidence and recommendations for future reports. J Neurol Neurosurg Psychiatry. 2004; 75: 1019-4. 
2. American College of Rheumatology. A Surgical Cure for Fibromyalgia and Chronic Fatigue? Reaction to an ABC News 20/20 Report. Available from: http://www.rheumatology.org/publications/hotline/0400fmsurgery. asp

3. Gutierrez-Alvarez AM, Beltran-Rodriguez J, Moreno CB. Antiepileptic drugs in treatment of pain caused by diabetic neuropathy. J Pain Symptom Manage. 2007; 34: 201-8.

4. Cepeda MS, Camargo F, Zea C, Valencia L. Tramadol for osteoarthritis. Cochrane Database Syst Rev. 2006; 3: CD005522.

5. Martell BA, O'Connor PG, Kerns RD, et al. Systematic review: opioid treatment for chronic back pain: prevalence, efficacy, and association with addiction. Ann Intern Med 2007; 146: 116-27.

6. Saarto T, Wiffen PJ. Antidepressants for neuropathic pain. Cochrane Database Syst Rev. 2005; 3: CD005454.

7. Casale R, Damiani C, Rosati V, et al. Efficacy of a comprehensive rehabilitation programme combined with pharmacological treatment in reducing pain in a group of OA patients on a waiting list for total joint replacement. Clin Exp Rheumatol. 2012; 30: 233-9.

8. Tremont-Lukats IW, Challapalli V, McNicol ED, et al. Systemic administration of local anesthetics to relieve neuropathic pain: a systematic review and meta-analysis. Anesth Analg 2005; 101: 1738-49.

9. Casale R, Tugnoli V. Botulinum toxin for pain. Drugs R D. 2008; 9: 11-27.

10. Bergman S. Management of musculoskeletal pain. Best Pract Res Clin Rheumatol. 2007; 21: 153-66.

11. Bruehl S, Chung OY. Psychological and behavioural aspects of complex regional pain syndrome management. Clin J Pain. 2006; 22: 430-7.

12. Morone NE, Greco CM. Mind-body interventions for chronic pain in older adults: a structured review. Pain Med. 2007; 8: 359-75.

13. Wall PD. The gate control theory of pain mechanisms. A re-examination and re-statement. Brain. 1978; 101: 1-18.

14. Heinricher MM, Tavares I, Leith JL, Lumb BM. Descending control of nociception: Specificity, recruitment and plasticity. Brain Res Rev. 2009; 60: 214-25.

15. Dacher M, Nugent FS Opiates and plasticity. Neuropharmacology. 2011; 61: 1088-96.

16. Buskila D. Genetics of chronic pain states. Best Pract Res Clin Rheumatol. 2007; 21: 535-47.

17. Gaumond I, Spooner MF, Marchand S. Sex differences in opioid-mediated pain inhibitory mechanisms during the interphase in the formalin test. Neuroscience. 2007; 146: 366-74.

18. Casale R, Cazzola M, Arioli G, et al. Italian Fibromyalgia Network. Non pharmacologi- cal treatments in fibromyalgia. Reumatismo 2008; 60 (Suppl. 1): 59-69.

19. Ceccherelli F, Gagliardi G, Visentin R, et al. The effects of parachlorophenylalanine and naloxone on acupuncture and electroacupuncture modulation of capsaicin-induced neurogenic oedema in the rat hind paw. A controlled blind study. Clin Exp Rheumatol. 1999; 17: 655-62.

20. Ceccherelli F, Altafini L, Varotto E, Stefecius A. The effect of benzodiazepines administration on auricular acupuncture symptomatological evidence. Acupunct Electrother Res. 1990; 15: 95-106.

21. Allen RJ. Physical agents used in the management of chronic pain by physical therapists. Phys Med Rehabil Clin N Am. 2006; 17: 31545.

22. Licciardone JC, Brimhall AK, King LN. Osteopathic manipulative treatment for low back pain: a systematic review and meta-analysis of randomized controlled trials. BMC Musculoskelet Disord. 2005; 6: 43-51.

23. Maigne R. The concept of painlessness and opposite motion in spinal manipulations. Am J Phys Med. 1965; 44: 55-69.

24. Twomey LT. A rationale for the treatment of back pain and joint pain by manual therapy. Phys Ther. 1992; 72: 885-92.

25. Haldeman S. Manipulation and massage for relief of back pain. In: R Melzack, PD Wall (eds.) Textbook of pain, 3rd ed. Churchill Livingstone, Edinburgh. 1994; 1251-62.

26. Fernandez-de-las-Penas C, Perez-de-Heredia M, Brea-Rivero M, Miangolarra-Page JC. Immediate effects on pressure pain threshold following a single cervical spine manipulation in healthy subjects. J Orthop Sports Phys Ther. 2007; 37: 325-9.

27. Benedetti F. Placebo and endogenous mechanisms of analgesia. Handb Exp Pharmacol. 2007; 177: 393-413.

28. Barron CJ, Moffett JA, Potter M. Patient expectations of physiotherapy: definitions, concepts, and theories. Physiother Theory Pract. 2007; 23: 37-46.

29. Casale R, Sommovigo B. Conventional use of the hands in physiotherapy. In: R Casale, F Ceccherelli, M Van Wassenhoven (eds), Unconventional medicine at the beginning of the third millennium. COST. B4, 68, 1998.

30. Sato A, Schmidt RF. Somatosympathetic reflexes: afferent fibers, central pathways, discharge characteristics. Physiol Rev. 1973; 53: 916-47.

31. Summerlee AJ, Lincoln DW. Electrophysiological recordings from oxytocinergic neurones during suckling in the unanaesthetized lactating rat. J Endocrinol. 1981; 90: 255-65.

32. Teirich-Leube H, Dicke E. Massage reflektorischen Zonen im Bindegewebe bei Rheumatischen und inneren Erkangkungen. Stuttgart, Gustav Fisher Verlag, 1942. 
33. LaMotte RH, Mountcastle VB. Capacities of humans and monkeys to discriminate vibratory stimuli of different frequency and amplitude: a correlation between neural events and psychological measurements. J Neurophysiol. 1975; 38: 539-59.

34. Melzack R. Acupuncture and related forms of folk medicine. In: PD Wall, R Melzack (eds) Textbook of pain, Edinburgh, Churchill Livingstone. 1984; 691-700.

35. Kagiki R, Shibasaki H. Mechanisms of pain relief by vibration and movement. J Neurol Neurosurg Psich. 1992; 55: 282-6.

36. Talbot WH, Darian-Smith I, Kornhuber HH, Mountcastle VB. The sense of flutter-vibration: comparison of the human capacity with response patterns of mechanoreceptive afferents from the monkey hand. J Neurophysiol. 1968; 31: 301-34.

37. Cosh JA. Studies on the nature of vibration sense. Clin Sci (Lond). 1953; 12: 131-51.

38. Casale R, Giordano A, Tiengo M. Spinal nociceptive reflex response: Changes in the RaIII nociceptive reflex response and in LBP induced by transcutaneous electric nerve stimulation and vibrations. Minerva Anestesiol. 1985; 51: 217-23.

39. Lundberg T, Nordemar R, Ottoson D. Pain alleviation by vibratory stimulation. Pain 1984; 20: $25-44$.

40. Casale R, Ring H, Rainoldi A. High frequency vibration conditioning stimulation centrally reduces myoelectrical manifestation of fatigue in healthy subjects. J Electromyogr Kinesiol. 2009; 19: 998-1004.

41. Casale R, Rainoldi A. Fatigue and fibromyalgia syndrome: clinical and neurophysiologic pattern. Best Pract Res Clin Rheumatol 2011; 25: 241-7.

42. Brown KE, Nickels FA, Caron JP, Mullineaux DR, Clayton HM. Investigation of the immediate analgesic effects of extracorporeal shock wave therapy for treatment of navicular disease in horses. Vet Surg. 2005; 34: 554-8.

43. Thomson CE, Crawford F, Murray GD. The effectiveness of extra corporeal shock wave therapy for plantar heel pain: a systematic review and meta-analysis. BMC Musculoskelet Disord. 2005; 6: 19-21.

44. Lehmann JF, deLateur BF. Ultrasound, shortwave, microwave, superficial heat and cold in the treatment of pain. In: PD Wall, R Melzack (eds) Textbook of pain. Edinburgh, Churchill Livingstone. 1984; 717-42.

45. LaMotte RH, Campbell JN. Comparison of responses of warm and nociceptive $\mathrm{C}$-fiber afferents in monkey with human judgments of thermal pain. J Neurophysiol. 1978; 41: 509-28.

46. Roelofs MF, Boelens WC, Joosten LA, et al. Identification of small heat shock protein B8 (HSP22) as a novel TLR4 ligand and potential involvement in the pathogenesis of rheuma- toid arthritis. J Immunol. 2006; 176: 7021-7.

47. French SD, Cameron M, Walker BF, et al. A Cochrane review of superficial heat or cold for low back pain. Spine. 2006; 31: 998-1006.

48. Rutkove SB. Effects of temperature on neuromuscular electrophysiology. Muscle Nerve. 2001; 24: 867-82.

49. Long WB 3rd, Edlich RF, Winters KL, Britt LD. Cold injuries. J Long Term Eff Med Implants. 2005; 15: 67-78.

50. Melzack R, Bentley KC. Relief of dental pain by ice massage of either hand or the contralateral arm. J Can Dent Assoc. 1983; 49: 257-60.

51. Melzack R, Jeans ME, Stratford JG, Monks $\mathrm{RC}$. Ice massage and transcutaneous electrical stimulation: comparison of treatment for lowback pain. Pain. 1980; 9: 209-17.

52. Carroll D, Moore RA, McQuay HJ, et al. Transcutaneous electrical nerve stimulation (TENS) for chronic pain. Cochrane Database Syst Rev. 2001; 3: CD003222.

53. Alfonsi E, Dalla Toffola E, Felicetti G, et al. Evaluation of the antidromic sensory conduction velocity of the sural nerve before and after antalgic Electrostimulation. G Ital Med Lav. 1982; 4: 239-40.

54. Casale R, Zelaschi F, Guarnaschelli C, Bazzini G. Electroanalgesia by transcutaneous stimulation (TNS). Response to the naloxone test. Minerva Med. 1983; 74: 941-6.

55. Maeda Y, Lisi TL, Vance CG, Sluka KA. Release of GABA and activation of GABA(A) in the spinal cord mediates the effects of TENS in rats. Brain Res. 2007; 1136: 43-50.

56. Hingne PM, Sluka KA. Differences in waveform characteristics have no effect on the anti-hyperalgesia produced by transcutaneous electrical nerve stimulation (TENS) in rats with joint inflammation. J Pain. 2007; 8: 251-5.

57. Gadsby J, Flowerdew M. WITHDRAWN: Transcutaneous electrical nerve stimulation and acupuncture- like transcutaneous electrical nerve stimulation for chronic low back pain. Update of Cochrane Database Syst Rev 2000; 2; CD000210. Cochrane Database Syst Rev. 2007; 3: CD000210.

58. Johnson MI, Tabasam G. An investigation into the analgesic effects of interferential currents and Transcutaneous Electrical Nerve Stimulation on experimentally induced ischemic pain in otherwise pain-free volunteers. Phys Ther. 2003; 83: 208-23.

59. Semalty A, Semalty M, Singh R, et al. Iontophoretic drug delivery system: A review. Technol Health Care. 2007; 1 5: 237-45.

60. Markov MS. Magnetic field therapy: a review. Electromagn Biol Med. 2007; 26: 1-23.

61. Carpenter DO, Ayrapetyan S. Biological effects of electric and magnetic fields: sources and mechanisms. San Diego, Academic Press. 1994. 
62. Woldanska-Okonska M, Karasek M, Czernicki J. The influence of chronic exposure to low frequency pulsating magnetic fields on concentrations of FSH, LH, prolactin, testosterone and estradiol in men with back pain. Neuro Endocrinol Lett. 2004; 25: 201-6.

63. Karu TI. The science of low power laser therapy. London, Gordon \& Breach Ed, 1998.

64. Karu TI, Pyatibrat LV, Afanasyeva NI. Cellular effects of low power laser therapy can be mediated by nitric oxide. Lasers Surg Med. 2005; 36: 307-14.

65. Jimbo K, Noda K, Suzuki K, Yoda K. Suppressive effects of low-power laser irradiation on bradykinin evoked action potentials in cultured murine dorsal root ganglion cells. Neurosci Lett. 1998; 240: 93-6.

66. Honmura A, Yanase M, Obata J, Haruki E. Therapeutic effect of Ga-Al-As diode laser irradiation on experimentally induced inflammation in rats. Lasers Surg Med. 1992; 12: 441-9.

67. Brosseau L, Robinson V, Wells G, et al. WITHDRAWN: Low level laser therapy (Classes III) for treating osteoarthritis. Update of Cochrane Database Syst Rev2004; 3: CD002046. Cochrane Database Syst Rev 2007; 3: CD002046.
68. Casale R, Damiani C, Maestri R, Wells CD. Pain and electrophysiological parameters are improved by combined 830-1064 high-intensity LASER in symptomatic carpal tunnel syndrome versus Transcutaneous Electrical Nerve Stimulation A randomized controlled study. Eur J Phys Rehabil Med 2012 (Epub ahead of print).

69. Batavia M. Contraindications for superficial heat and therapeutic ultrasound: do sources agree? Arch Phys Med Rehabil. 2004; 85: 1006-12.

70. Mann T, Refshauge KM. Causes of complications from cervical spine manipulation. Aust J Physiother. 2001; 47: 255-66.

71. Casale R, Gibellini R, Bozzi M, Bonelli S. Changes in sympathetic activity during high frequency T.E.N.S. Acupunct Electrother Res. 1985; 10: 169-75.

72. Bjordal JM, Bogen B, Lopes-Martins RA, Klovning A. Can Cochrane Reviews in controversial areas be biased? A sensitivity analysis based on the protocol of a Systematic Cochrane Review on low-level laser therapy in osteoarthritis. Photomed Laser Surg. 2005; 23: 453-8 